\title{
Reaction Wheel Disturbance Modeling, Jitter Analysis, and Validation Tests for Solar Dynamics Observatory
}

\author{
Kuo-Chia Liu ${ }^{1}$ and Peiman Maghami ${ }^{2}$ \\ NASA Goddard Space Flight Center, Greenbelt, MD, 20771 \\ Carl Blaurock ${ }^{3}$ \\ Nightsky Systems Inc, Raleigh, NC, 27616
}

\begin{abstract}
The Solar Dynamics Observatory (SDO) aims to study the Sun's influence on the Earth by understanding the source, storage, and release of the solar energy, and the interior structure of the Sun. During science observations, the jitter stability at the instrument focal plane must be maintained to less than a fraction of an arcsecond for two of the SDO instruments. To meet these stringent requirements, a significant amount of analysis and test effort has been devoted to predicting the jitter induced from various disturbance sources. One of the largest disturbance sources onboard is the reaction wheel. This paper presents the SDO approach on reaction wheel disturbance modeling and jitter analysis. It describes the verification and calibration of the disturbance model, and ground tests performed for validating the reaction wheel jitter analysis. To mitigate the reaction wheel disturbance effects, the wheels will be limited to operate at low wheel speeds based on the current analysis. An on-orbit jitter test algorithm is also presented in the paper which will identify the true wheel speed limits in order to ensure that the wheel jitter requirements are met.
\end{abstract}

\section{Nomenclature}

$C \quad=$ amplitude coefficient of wheel disturbance

$c_{a x} \quad=$ damping of wheel axial mode

$c_{\text {rock }}=$ damping of wheel rocking mode

$d \quad=$ wheel disturbance input

$h \quad=$ harmonic number

$F_{\text {rad }}=$ wheel radial force disturbance

$F_{x}, F_{y} \quad=\mathrm{X}, \mathrm{Y}$ component of wheel disturbance force

$F_{z} \quad=\mathrm{Z}$ component of wheel disturbance force (axial force, $F_{z}=F_{a x}$ )

$G_{z w}=$ Transfer function from wheel disturbance input to performance output

$I_{r r} \quad=$ Wheel inertial perpendicular to spin axis

$I_{z z} \quad=$ Wheel inertial about spin axis

$k_{a x} \quad=$ stiffness of wheel axial mode

$k_{\text {rock }}=$ stiffness of wheel rocking mode

$M \quad=$ Wheel mass

$M_{\text {rad }} \quad=$ wheel radial moment disturbance

$M_{x}, M_{y}=\mathrm{X}, \mathrm{Y}$ component of wheel disturbance torque

$M_{z} \quad=\mathrm{Z}$ component of wheel disturbance torque

$\Omega \quad=$ Wheel speed

$\phi \quad=$ Phase angle

$S \quad=\quad$ Power spectral density (PSD)

$S_{w w} \quad=$ Reaction wheel disturbance PSD

${ }^{1}$ SDO Jitter Lead Analyst, Guidance, Navigation, and Control Systems Engineering Branch, Code 591, and AIAA Lifetime Member.

${ }^{2}$ SeniorAnalyst, Guidance, Navigation, and Control Systems Engineering Branch, Code 591, and AIAA Member.

${ }^{3}$ President, 3916 Lauriston Rd., Raleigh NC 27616, and AIAA Member. 


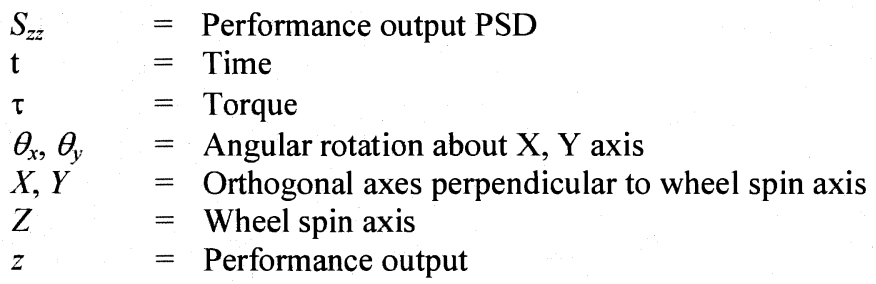

\section{Introduction}

$\mathrm{T}$ he Solar Dynamics Observatory (SDO) is scheduled for launch in January 2009. The main science objective of this mission is to observe the Sun and increase the understanding of the Sun's influence on the Earth. There are three instruments onboard the SDO for achieving its scientific goals: the Atmospheric Imaging Assembly (AIA), the Helioseismic and Magnetic Imager (HMI), and the Extreme Ultraviolet Variability Experiment (EVE). Both AIA and HMI are sensitive to high frequency pointing perturbations and have tight jitter requirements. The SDO jitter performance is defined as the line-of-sight (LOS) motion measured at the instrument detectors. The spacecraft attitude control system (ACS) is capable of removing large, slowly varying LOS (or pointing) errors. Both AIA and HMI have additional instrument stabilization systems (ISS) to reduce mid-frequency range LOS disturbances. The residual LOS motion, after ACS and ISS compensation, measured on the instrument detectors must be less than 0.17 arcsecond $1-\sigma$ for AIA and 0.14 arcsecond 1- $\sigma$ for HMI.

One of the largest disturbance sources onboard the spacecraft is the reaction wheel. SDO carries four wheels to provide 3-axis pointing stabilization. These wheels also generate large tonal and broadband disturbances due to mass imbalances, bearing imperfections, and mechanical noises. The wheel disturbances are difficult to model accurately since their frequency and magnitude change with wheel speeds, and since they can interact with wheel structural modes to greatly amplify the disturbance level. The wheel jitter allocations from the total AIA and HMI jitter requirements are 70 milliarcsecond (mas) and 62 mas, respectively. The tight jitter requirements necessitate careful modeling of the wheel disturbance and validation of the analysis results. The uncertainties inherent in predicting wheel disturbances and structural response also motivate the development of a test plan to measure jitter on-orbit and adjust wheel operational parameters accordingly.

Extensive modeling and analysis efforts have been directed in estimating the amount of jitter disturbing the science instruments. This paper first describes our approach on wheel disturbance modeling in Section II. It then presents how the disturbance model is used to predict jitter performance by using an integrated modeling methodology in Section III. In order to verify the disturbance models and to validate the jitter performance prior to launch, the wheel induced vibration levels were tested by the wheel vendor, and a close-to observatory level wheel disturbance test was performed at the NASA Goddard Space Flight Center (GSFC). This paper provides descriptions of the test setup and relevant results from the ground tests in Section IV. Finally, the paper also presents SDO's onorbit jitter test plans and wheel disturbance test algorithms in Section V.

\section{Reaction Disturbance Model}

SDO has four Goodrich E-type reaction wheels onboard the spacecraft for attitude control. These wheels are mounted on the four upper bus panels of the spacecraft with the spin axis pointing 60 deg away from the body $\mathrm{X}$ axis. The maximum torque capability of each wheel is $0.25 \mathrm{Nm}$ with a momentum limit of $70 \mathrm{Nms}$. The reaction wheels are the source of high amplitude jitter disturbance, due to static imbalance, dynamic imbalance, and bearing imperfections that introduce tonal disturbances occurring at known ratios of the wheel speed. In addition, the noise signature includes a low level broadband noise characteristic that is visible at low wheel speeds. Since the net solar and gravitational torques acting on SDO are expected to be low, the wheel speed change will be very slow. As a result, the wheel tonal disturbances will dwell on jitter-critical observatory modes long enough to excite them to steady state. The modeling assumptions and the wheel disturbance model are described in this section.

The goal of this section is to present the wheel disturbance modeling methodology used by the SDO analysis team. The precise disturbance level is not crucial to this discussion. In order to protect the proprietary wheel disturbance data provided by the manufacturer, several plots shown in this section have magnitudes purposefully blanked out. 


\section{A. Model Assumptions}

The reaction wheel Induced Vibration (IV) disturbance forces were measured by Goodrich in order to verify the imbalance requirements. Figure 1 shows a typical data set for one SDO wheel (post-vibration). The plot shows the force in the wheel $\mathrm{Y}$ axis, as a function of frequency and wheel speed in revolutions per second (rev/sec). Note that the forces and torques are defined at the wheel center of mass. The ridge lines fanning diagonally out from the lower left corner are referred to as tonal.disturbances. These are wheel dependent disturbances occurring at frequencies equal to harmonics of the wheel speed.

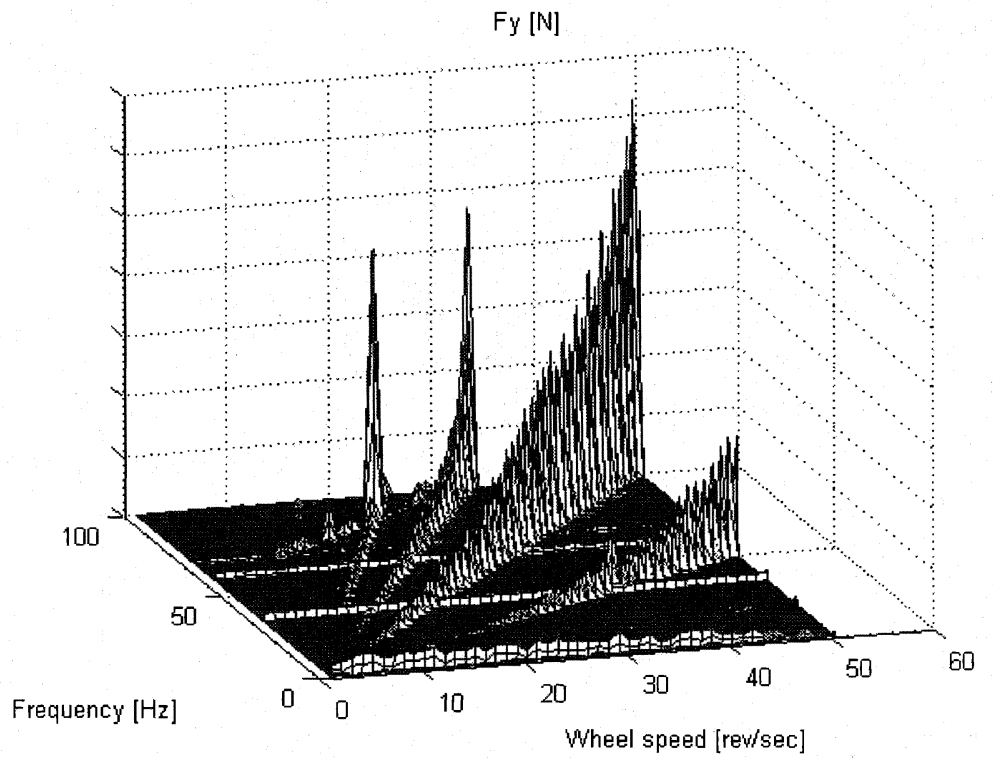

Figure 1 Wheel disturbance Induced Vibration (IV) data for one SDO wheel, in the $Y$ axis, as a function of frequency and wheel speed

The IV test data sets could be used to perform the jitter analysis directly. However, the disturbance data were taken at a relatively coarse set of wheel speeds, in 60 revolutions per minute (RPM) steps. The tonal disturbances from the discrete data sets are unlikely to occur exactly at the frequency of spacecraft structural modes, and therefore the peak jitter will be missed. As an alternative, a semi-analytical wheel disturbance model was created and tuned to the test data. The model was then used to predict the disturbances at a very fine wheel speed resolution, to ensure that the peak response was captured.

The semi-analytical wheel model consisted of a physical model of the wheel axial and rocking modes, including gyroscopic torques on the wheel, excited by external forces. In addition to tonal disturbances, broadband noise was another distinct forcing characteristic visible from the test data. Analysis results described later in the paper demonstrate that both tonal and broadband disturbances were important. The tonal disturbances were larger in magnitude and created the largest jitter response when aligned with the spacecraft modes, but the broadband noise was always present and therefore continuously pumped energy into wheel structural modes, producing a continual jitter response at all wheel speeds. The semi-analytical disturbance model is diagrammed in Figure 2. It consisted of empirical tonal and broadband disturbance models, generated from fits to the disturbance data. The disturbances were filtered by an analytical model of the reaction wheel structural modes, which accounted for the dynamic amplification of the disturbances at wheel structural frequencies. The model development proceeded by, first, identifying the harmonic disturbances and tuning the wheel structural modes, since the higher disturbance magnitude produced a higher signal to noise. Then the harmonics were removed from the disturbance data to produce a broadband noise model. The tonal disturbance model, wheel structural model, and broadband noise model are discussed below. 


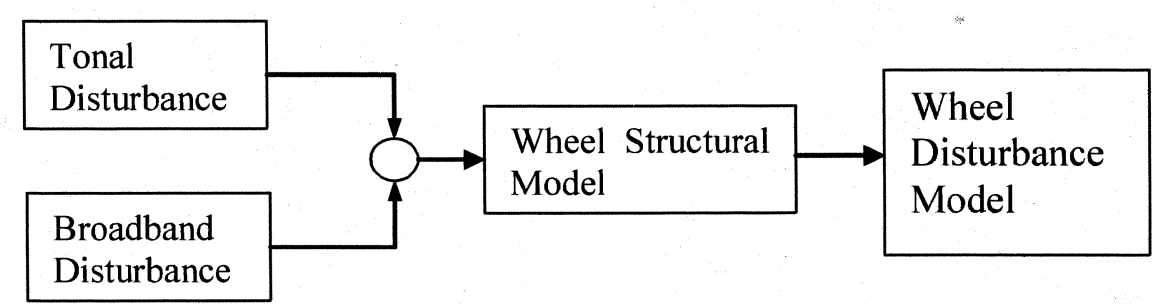

Figure 2 Semi-analytical wheel model consists of empirical tonal and broadband disturbance models, filtered by an analytical model of $R W$ dynamics

\section{B. Wheel Tonal Disturbance Model}

In the reaction wheel frame, the positive spin axis defines the local $Z$ axis, and any two orthogonal vectors perpendicular to spin axis form the $X$ and $Y$ axes. The disturbance forces generated in the wheel plane are referred to as radial forces $\left(F_{x}, F_{y}\right)$, force along the $Z$ axis is the axial force $\left(\mathrm{F}_{z}\right)$, torques around $X$ and $Y$ axes are radial torques $\left(M_{x}, M_{y}\right)$, and the torque around the spin axis is the axial torque $\left(M_{z}\right)$. Test data have shown that wheel imbalance and bearing imperfections only result in small disturbance torques around the spin axis; therefore, axial torque is neglected for the wheel disturbance modeling and jitter analysis.

The forcing characteristics were modeled by a speed-squared model for the tonal disturbance ${ }^{1}$ :

$$
d_{j}(t)=\sum_{k=1}^{N_{j}} C_{j k} \Omega^{2} \sin \left(2 \pi h_{j k} \Omega t+\phi_{j k}\right)
$$

where $d$ is disturbance input, $j$ is disturbance input number (for force, $j=1,2,3$, or for torque, $j=4,5,6$ ), $k$ is the harmonic number index, $N_{j}$ is the number of harmonics for $j^{\text {th }}$ disturbance input, $C_{j k}$ is the amplitude coefficient for $k^{\text {th }}$ harmonic of $j^{\text {th }}$ disturbance input, $\Omega$ is the wheel speed in $\mathrm{Hz}, h_{j k}$ is the harmonic number (ratio of frequency of $k^{\text {th }}$ harmonic of $j^{\text {th }}$ disturbance to spin frequency of the wheel), and $\phi_{j k}$ is the phase angle of $k^{\text {th }}$ harmonic of $j^{\text {th }}$ disturbance. Each disturbance component is the sum of sinusoids occurring at various harmonic frequencies, $h_{j k} \Omega$.

In Eq. (1), the parameters $C_{j k}$ and $h_{j k}$ are determined or calibrated from the IV test data. The procedure will be described in Section D. The phase angles $\phi_{j k}$ are assumed to be random with uniform distribution between 0 and $2 \pi^{1}$.

\section{Wheel Structural Model}

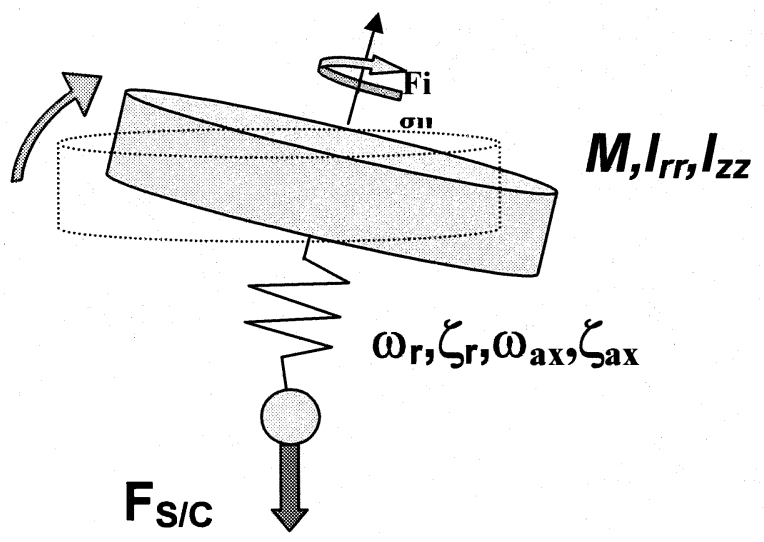

Figure 3 Schematic of the wheel structural model

The gyroelastic wheel structural model accounts for elastic deformation of the wheel, shaft, bearings, and case, as acted on by gyroscopic forces created by, and applied to, the spinning wheel. This deformation is important to the jitter response because it dynamically amplifies the disturbance force, by as much as a factor of 50 for the SDO wheels. The wheel structure is modeled by a simplified, but physically meaningful dynamic system (Figure 3 ). The model includes wheel rocking about the two axes perpendicular to spin, and axial displacement along the spin axis. 
Wheel disturbance forces are applied at the center of mass (CM), where they are filtered by the structural modes to generate dynamically amplified disturbances. The Goodrich IV data shows no evidence of modes in the lateral force data, or in the spin axis torque, so these three axes are modeled as a pass-through of external disturbances directly to the spacecraft.

Note that the offset from wheel CM to wheel mounting interface will cause the lateral disturbance forces to create a moment at the interface. The wheel disturbance node in the FE model is located at the wheel CM, so that the wheel CM disturbances will cause the correct moments.

The model parameters for the wheel structural model include the mass $M$, inertias of the wheel about the spin axis, $I_{z z}$, and about its perpendicular, $I_{r r}$, the wheel rocking mode frequency $\omega_{\mathrm{r}}$ and damping $\zeta_{\mathrm{r}}$ at zero RPM, and the axial mode frequency $\omega_{\mathrm{ax}}$ and damping $\zeta_{\mathrm{ax}}$. Mass, inertia, and frequency parameters are provided by the wheel manufacturer. They can be verified, and if necessary tuned, using the IV data. The damping parameters must be extracted from the IV data to account for any possible amplitude dependence.

The rocking and axial dynamics can be treated independently. The rocking dynamics are a function of the rotational inertia of the wheel perpendicular to the spin axis, $I_{r r}$, the effective stiffness of the wheel, shaft, bearings, and case, $K_{\text {rock }}$, as well as the effective damping $C_{r o c k}$, and the gyroscopic torque in terms of spin axis inertia $I_{z z}$ and the wheel spin rate $\Omega^{2}$,

$$
\left[\begin{array}{cc}
I_{r r} & 0 \\
0 & I_{r r}
\end{array}\right]\left[\begin{array}{c}
\ddot{\theta}_{x} \\
\ddot{\theta}_{y}
\end{array}\right]+\left[\begin{array}{cc}
c_{r o c k} & 0 \\
0 & c_{r o c k}
\end{array}\right]\left[\begin{array}{c}
\dot{\theta}_{x} \\
\dot{\theta}_{y}
\end{array}\right]+\Omega\left[\begin{array}{cc}
0 & I_{z z} \\
-I_{z z} & 0
\end{array}\right]\left[\begin{array}{c}
\dot{\theta}_{x} \\
\dot{\theta}_{y}
\end{array}\right]+\left[\begin{array}{cc}
k_{\text {rock }} & 0 \\
0 & k_{\text {rock }}
\end{array}\right]\left[\begin{array}{l}
\theta_{x} \\
\theta_{y}
\end{array}\right]=\left[\begin{array}{l}
\tau_{x} \\
\tau_{y}
\end{array}\right]
$$

The torques on the spacecraft are computed from the differential angular rotation of the wheel,

$$
\left[\begin{array}{l}
\tau_{x} \\
\tau_{y}
\end{array}\right]_{S / C}=\left[\begin{array}{cc}
k_{r o c k} & 0 \\
0 & k_{r o c k}
\end{array}\right]\left[\begin{array}{l}
\theta_{x} \\
\theta_{y}
\end{array}\right]
$$

The effective stiffness and damping can be computed from the rocking mode frequency and damping ratio at zero RPM,

$$
\begin{aligned}
& k_{\text {rock }}=I_{r r}\left(2 \pi f_{\text {rock }}\right)^{2} \\
& c_{\text {rock }}=4 \zeta_{\text {rock }} f_{\text {rock }} I_{r r}
\end{aligned}
$$

As noted, these parameters are provided by the wheel manufacturer but can be tuned during model calibration.
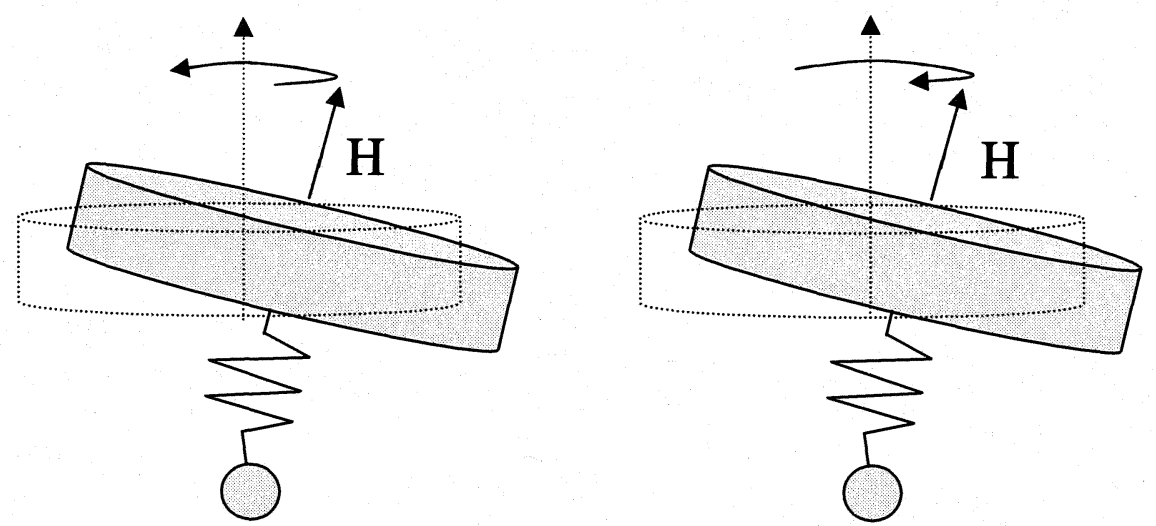

Figure 4 (left) nutational mode (posigrade): the angular momentum vector tip describes a circle, in the same direction as the wheel rotation, (right) nutational mode (retrograde): the tip describes a circle in the opposite direction to the rotation 
The gyroscopic terms couple the rocking modes. At zero RPM, there are two orthogonal modes at the same frequency. When the wheel spins, the modes couple and split into a nutational and a precessional mode, collectively called whirl modes. These modes can be visualized in terms of the angular momentum vector $\mathrm{H}$ (Figure 4). At zero RPM, when a rocking mode is excited, the tip of the vector oscillates in a single plane. As RPM increases, the tip of the vector starts to move in a circle. The nutational mode involves the tip moving in the same direction as the spin (posigrade motion). This mode increases in frequency as the spin rate rises. The precessional mode involves the tip moving in the opposite sense to the spin direction (retrograde), and this mode drops in frequency as spin rate rises. Thus the rocking mode splits into two "branches" as spin rate increases (Figure 5$)^{3}$.

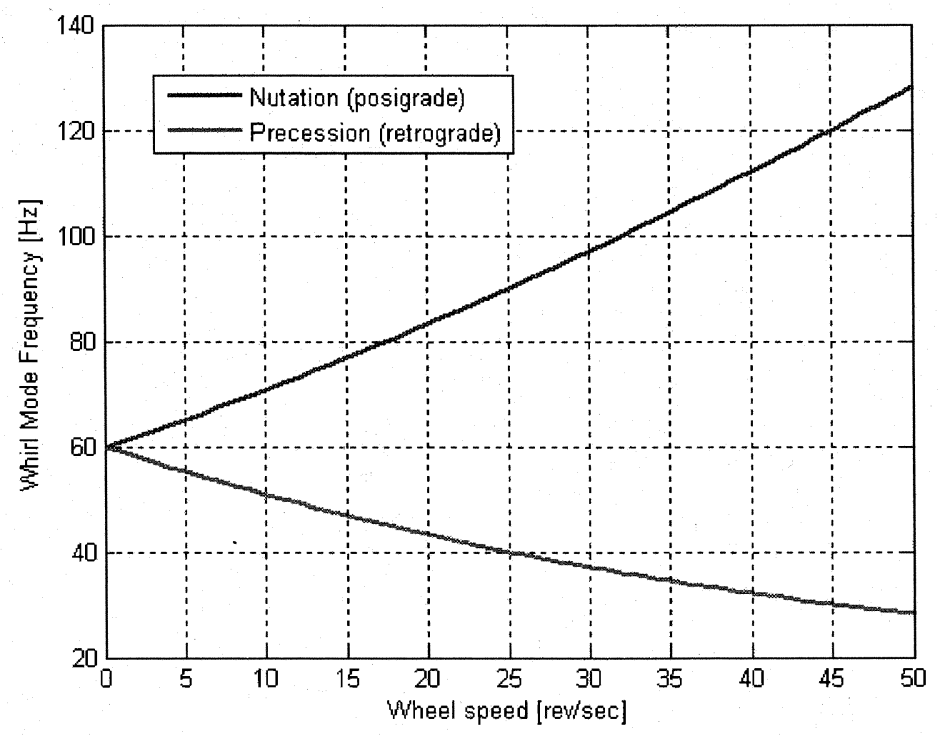

Figure 5 Goodrich $\mathrm{E}$ whirl modes start at $60 \mathrm{~Hz}$ rock mode. Nutation branch increases with frequency, precession branch decreases with frequency.

The axial force model is not wheel speed dependent, and can therefore be defined simply in terms of a second order dynamic model,

$$
\begin{aligned}
& M \ddot{Z}+c_{a x} \dot{Z}+k_{a x} Z=f_{z} \\
& f_{z, S C}=k_{a x} Z
\end{aligned}
$$

where $M$ is the wheel mass, $\mathrm{c}_{\mathrm{ax}}$ is the damping, $\mathrm{k}_{\mathrm{ax}}$ is the effective stiffness, and the latter two parameters can be computed from the measured frequency and damping ratio,

$$
\begin{aligned}
& k_{a x}=M\left(2 \pi f_{a x}\right)^{2} \\
& c_{a x}=4 \zeta_{a x} f_{a x} M
\end{aligned}
$$

Similarly to the rock mode, these parameters can be refined during model calibration.

The wheel gyroscopic terms require careful treatment of disturbance phasing. The radial forces and moments are correlated. The static and dynamic forces and torques, in particular, have a known phase. For a positive spin, the Y force/moment lags the $\mathrm{X}$ force/moment by 90 degrees. For negative spin, the $\mathrm{Y}$ forces/moments lead. For higher harmonics, the phase relationship is not known a priori so it must be extracted from the IV data. In the frequency domain, the relationship from radial forces and moments to $\mathrm{X} / \mathrm{Y}$ forces and moments is: 


$$
\left[\begin{array}{c}
F_{x} \\
F_{y} \\
F_{z} \\
M_{x} \\
M_{x}
\end{array}\right]=\left[\begin{array}{ccccc}
1 & 1 & & & \\
-i & i & & & \\
& & 1 & & \\
& & & 1 & 1 \\
& & & -i & i
\end{array}\right]\left[\begin{array}{c}
F_{r a d+} \\
F_{r a d-} \\
F_{z} \\
M_{r a d+} \\
M_{r a d-}
\end{array}\right]
$$

where $\mathrm{i}=$ sqrt(-1) and the subscripts on the right hand side refer to the wheel spin direction. The phasing matrix can be folded into the analysis model. The disturbance fundamental is caused by static and dynamic imbalance, which has physically a + spin phase relationship, so the jitter response is evaluated from $F_{r a d}, F_{z}$, and $M_{r a d}$. For higher harmonics, the root-sum-square (RSS) of the +spin and -spin responses should be used. For the SDO wheel jitter analysis, the computations were simplified slightly by RSS'ing the +spin and -spin forces and moments prior to impinging them on the structural model. This simplification matched the IV data well.

The approach used for SDO was to evaluate the disturbance forces on the fixed-base wheel model, and apply these to the spacecraft structural model. This technique ignored the coupling between wheel and spacecraft structural dynamics, which could result in a shift in rocking and axial modes. The alternative of incorporating the wheel model into the FEM, and applying the gyroscopic terms to the FEM during the jitter analysis, would be more accurate but substantially more computationally intensive. In practice, the simplification was unlikely to lead to under-predicting the peak jitter since the analysis allowed for a $+/-10 \%$ structural mode frequency shift to account for modeling uncertainty, and therefore the worst-case coupling between wheel disturbances and observatory structural modes should have been captured.

\section{Calibration Procedures for Tonal Disturbances and Structural Modes}

The reaction wheel disturbance model was calibrated to match the Goodrich IV data. Model parameters such as harmonic numbers $\left(h_{j k}\right)$ and amplitudes $\left(C_{j k}\right)$, and reaction wheel structural modal frequencies were calibrated to the induced vibration data.

All four SDO reaction wheels were tested, both before and after vibration testing. The post-vib data was used to calibrate four separate models. The calibration process proceeded by computing the amplitude spectra (AS) of the time domain wheel disturbance data to form waterfall data, as shown in Figure 1. The data sets consisted of 50 seconds of data at a sample rate of 800 samples per second. The intent was to use 5 averages of 10 seconds each to reduce noise, but the wheel speed drifted during the test. As a result, a single average of 10 seconds $(8,000$ samples $)$ was used. Since the analysis was completed, a technique for accommodating wheel drift has been developed.

In order to calibrate the harmonic model, the waterfall data sets were converted to order analysis data, consisting of amplitude as a function of harmonic factor $h$ and wheel speed $\Omega$. The harmonics were identified as constant-h ridgelines. Then, for each harmonic, the disturbance coefficients and wheel structural frequency and damping were simultaneously tuned to the data using a nonlinear least-squares technique.

As an example, Figure 6 plots the torque contours about the Y axis, for one SDO wheel. The dashed lines fanning out from the lower left are the identified harmonics, and the solid black lines are the whirl modes as predicted by the model. The peak disturbance occurs at several wheel speeds; at high wheel speed (above 35 $\mathrm{rev} / \mathrm{sec}$ ) at the fundamental, at $23 \mathrm{rev} / \mathrm{sec}$ when the second harmonic crosses the precessional branch, and at 36 $\mathrm{rev} / \mathrm{sec}$ when the $3 \mathrm{rd}$ harmonic crosses the nutational branch. The model captures the whirl mode behavior well at all wheel speeds. The broadband noise from 210 to $400 \mathrm{~Hz}$ is particularly evident in the data set. The continuous ridge lines tracing out the whirl mode branches in the data make it clear that the wheel structural modes are constantly excited by broadband energy. Figure 7 shows the comparison between the radial moment amplitude data, in blue, and the model fit, in green, for the fundamental harmonic $(\mathrm{h}=1.0)$. The prediction is almost exact between three and $20 \mathrm{rev} / \mathrm{sec}$. Below $3 \mathrm{rev} / \mathrm{sec}$ the data is dominated by noise. Above $20 \mathrm{rev} / \mathrm{sec}$, the model over-predicts the disturbance amplitude. The effect is due to the technique used at the time to convert the AS into order data, which could miss some of the disturbance energy and thus under-estimate the forcing magnitude. An improved order analysis method has since been developed that computes order data from power spectra, and which integrates energy over several frequency points to reduce this effect. 


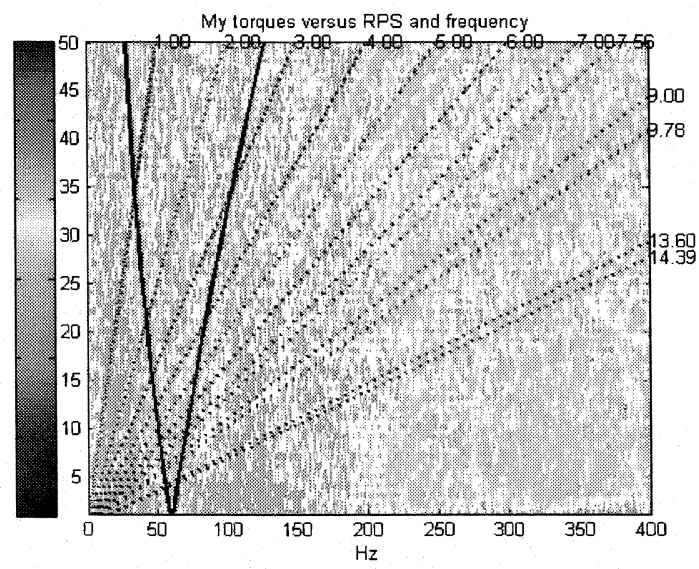

Figure 6 Contour plot of wheel torque (My)

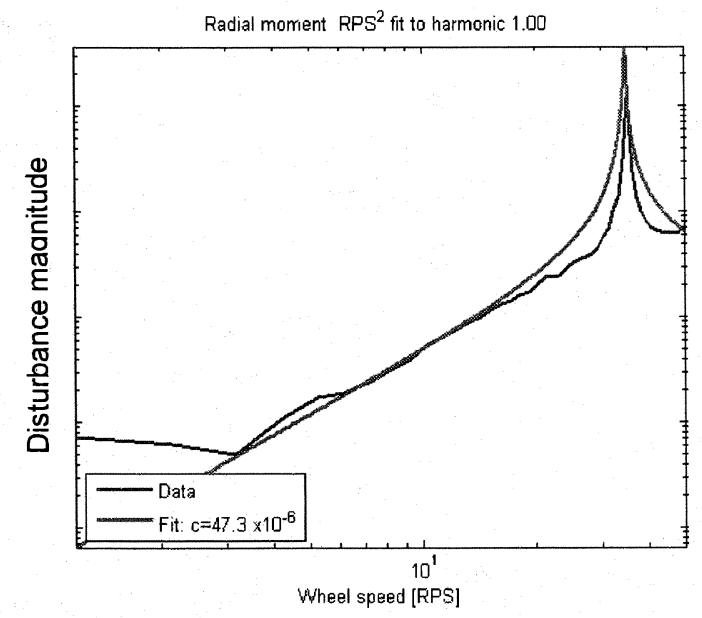

Figure 7 Radial moment fit to first harmonic

Each of the four wheel data sets was calibrated to produce four models, each having radial force, axial force, and radial moment coefficients. Between 12 and 23 harmonics from 0.4 to 14.54 times the fundamental were identified, depending on wheel and forcing axis. Wheel to wheel variability was significant. The four wheels had many of the same lower harmonics (to about 9.0 times the fundamental), but each wheel also had many unique harmonics. Above 9.0 times the fundamental, there were significantly more unique harmonics. The forcing levels at common harmonics varied significantly between wheels, by up to a factor of 10 .

\section{E. Wheel Broadband Disturbance Model}

Another significant wheel disturbance characteristic was broadband noise. Although typically not as large in magnitude as the tonal disturbances, it was present at low wheel speeds and introduced large jitter when amplified by wheel structural modes. The procedure for obtaining the broadband disturbance data was:

- Calculate the Power spectral density (PSD) from the time-domain wheel disturbance data $(Q(t))$

- After wheel tonal disturbances are identified, the PSD $\left(S_{Q}(\omega)\right)$ associated with the tonal disturbance frequencies was zeroed.

- Zeroed elements in the PSD data were replaced with local background noise by averaging 10 points before and after the zeroed PSD points.

- Repeat above procedure for each set of wheel speed data and for all four wheels

Figure 8 shows an example of the axial force PSD when the wheel is running at $850 \mathrm{rev} / \mathrm{min}$ (RPM). In this plot, the PSD raw data and the broadband noise PSD data are shown as a solid line and dashed-dotted line, respectively. A simple straight-line + second order filter model (shown as dashed line) was developed for describing the broadband noise. However, the second order model parameters varied greatly between wheel speed data sets, so it was difficult to extrapolate noise model parameters between the various wheel data sets. As a result, the broadband noise data, not an analytical model, was used directly for the wheel jitter analysis. This decision was justified on the basis that the energy was spread across a relatively broad frequency band (several frequency samples), in contrast to the tonal disturbances which had a very narrow bandwidth.

In Figure 8 the noise data is obtained after removing the tonal disturbances. There are two large peaks in the noise data: one is around $75 \mathrm{~Hz}$ which corresponds to the wheel axial mode, and the second one is a broader peak between 200 and $400 \mathrm{~Hz}$. The rise at high frequency is not due to test stand modes or wheel structural modes since the width of the rise is too broad. The physical phenomenon which causes the broadband noise is not well understood, although it is postulated to be a pseudo-random process such as rattling of the bearings as they travel. However, analysis shows that the LOS performance is not sensitive to the high frequency noise; it is most sensitive to broadband noise that is amplified by the wheel structural modes (axial and whirl modes).

A similar PSD plot for the radial moment disturbance at 850 RPM is illustrated in Figure 9. The dominant disturbance in the noise data came from the wheel mechanical noise exciting the wheel rocking modes ( $\sim 48$ and $\sim 76$ $\mathrm{Hz}$ at $850 \mathrm{RPM})$. Frequencies of these modes vary with wheel speeds as shown in Figure 5. 


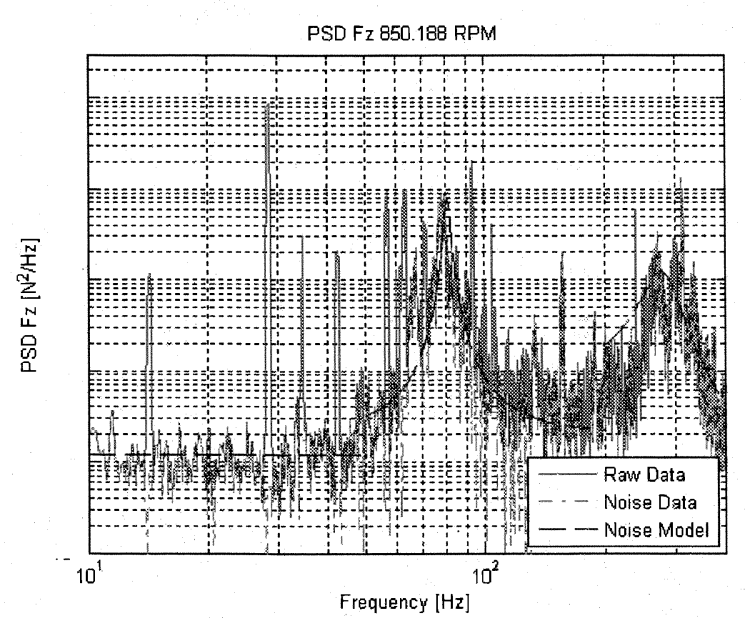

Figure 8 Broadband noise data for axial force disturbance

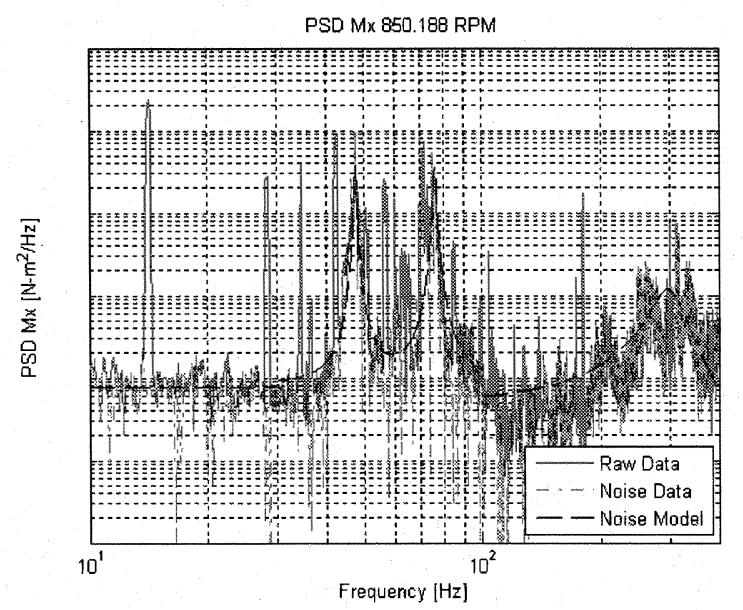

Figure 9 Broadband noise data for radial moment disturbance

\section{F. Disturbance Model Validation}

The wheel disturbance model was validated by computing the RMS energy versus wheel speed for the model and data, and ensuring that the model over-bounded the energy in the data. The comparison for a typical model is shown below. Figure 10 shows the radial force ( $\mathrm{X}$ and $\mathrm{Y}$ axes) compared to the model $\mathrm{X}$ axis force. The model overbounds the data at most wheel speeds. Below $7 \mathrm{rev} / \mathrm{sec}$, the $\mathrm{X}$ axis force is higher than the data, but the $\mathrm{Y}$ axis data is lower so the model is accurate to within the measurement noise. At $33 \mathrm{rev} / \mathrm{sec}$, the $\mathrm{h}=3$ harmonic crosses an unmodeled fixture mode that causes an extraneous peak in the forcing data. The axial force plot (Figure 11) shows that the model provides a fairly tight upper bound to the measured axial disturbance. The radial moment plot (Figure 12) again shows that the model provides an upper bound at all wheel speeds except for a small range 21 rev/sec. The results demonstrate that the wheel disturbance model provides a good estimate of the wheel induced forcing levels.

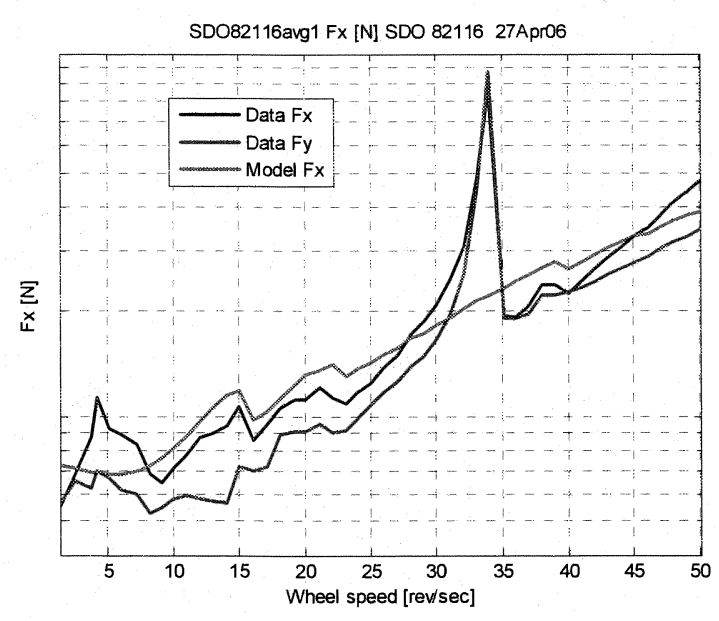

Figure 10 Axial force energy vs. wheel speed

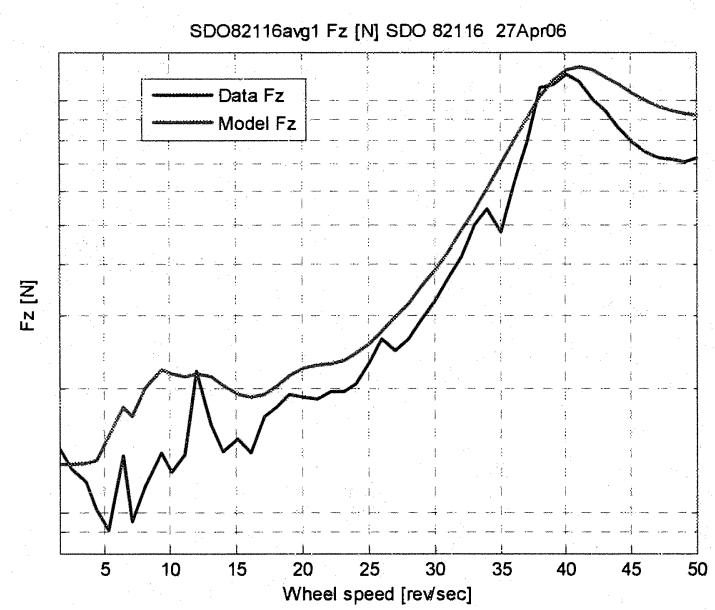

Figure 11 Radial force energy vs. wheel speed 


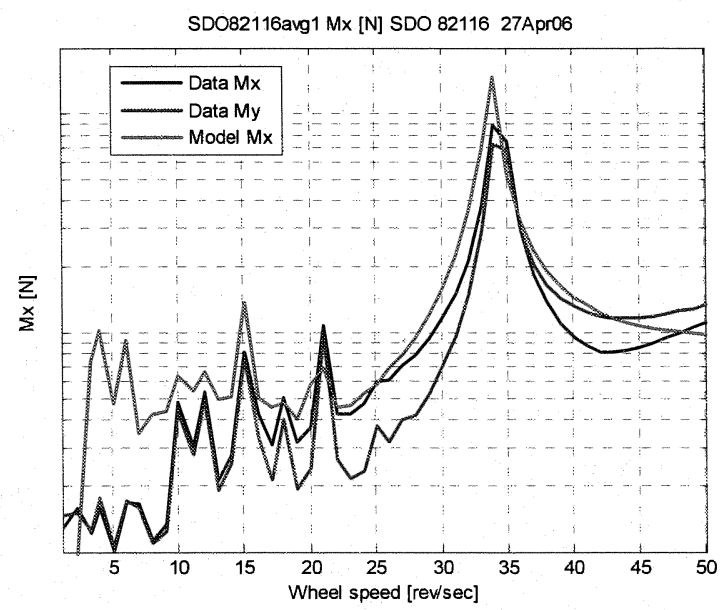

Figure 12 Radial moment energy vs. wheel speed

\section{Wheel Jitter Analysis}

The SDO jitter analysis employed an integrated modeling approach where disturbance, structures, controls, and performance metrics were combined into one dynamic model to predict the end-to-end LOS performance of the system. Figure 13 is a functional diagram of all the sub-system models used in the integrated model. The disturbance models were inputs to the system, the structural model was created from the finite element analysis, the ACS model used to stabilize the rigid body modes of the structural model, the optical model mapped motions of the optics to LOS, and the instrument stabilization system (ISS) modeled the LOS motion attenuation from the instrument controllers. The outputs from the integrated model were the LOS motion measured at the AIA and HMI charged coupled devices (CCDs).

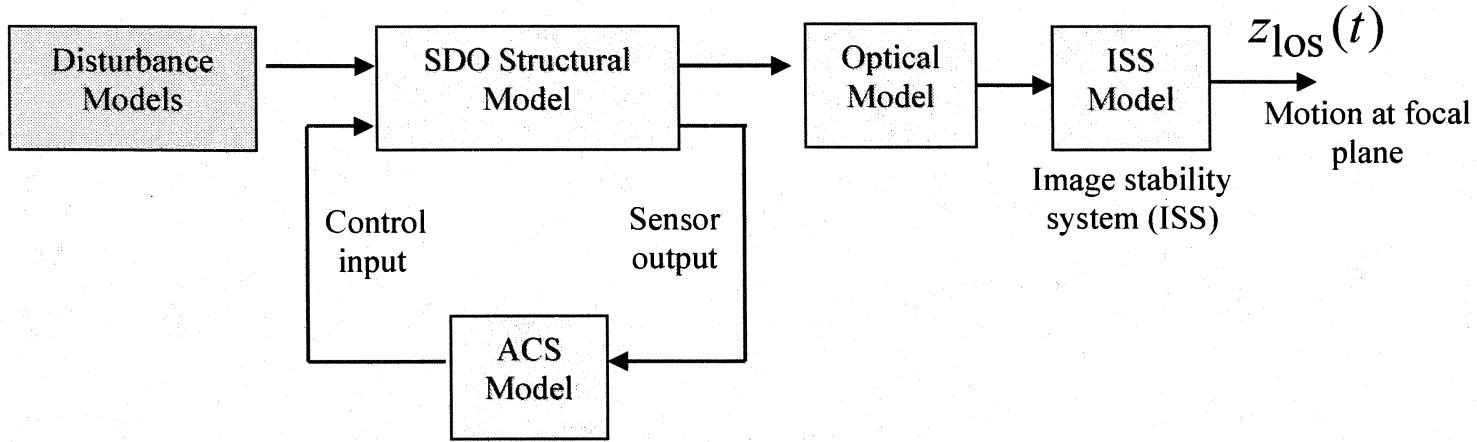

Figure 13 SDO integrated model

Details of the wheel disturbance model are described in the previous section. A brief description of the other models used in the integrated modeling analysis, as well as the analysis methods used to predict the LOS motion measured at the CCD are provided in this section. For more information on these models and how they are verified and validated, see Ref. 4.

\section{A. Integrated Model Descriptions}

Structural Model: The SDO FEM was developed to provide observatory modal information for use with advanced jitter analytical tools. The FEM represented the SDO in an on-orbit configuration: fully deployed, instrument covers open, and with $25 \%$ of the fuel and oxidizer mass remaining. The FEM included the spacecraft primary structure (Propulsion Module, Spacecraft Bus, and Instrument Module), spacecraft secondary structure (High Gain Antenna System (HGAS), Solar Array System (SAS) and hardware mounting brackets), and detailed instrument models (AIA, HMI, and EVE). Figure 14 shows the SDO jitter model. The normal modes solution of MSC/NASTRAN was run on the unconstrained FEM, and mode shape and frequency data for a subset of 120 nodes was used in the jitter analysis. The model had over 650 modes between 0 and $200 \mathrm{~Hz}$. 
Damping Model: The damping model assumed uncoupled modal damping. The scalar damping value, used for all modes, was calibrated by test. The test article which consisted of the SDO structural verification unit was excited using a proof mass actuator through a force-gage-equipped stinger. Frequency Response Functions (FRFs) from input force to 19 accelerometer responses were acquired with a DataMax data acquisition system. The FRFs were fit with system identification tools to produce a total of 88 Single Input, Multiple Output (SIMO) state space models representing different amplitudes, input locations, and accelerometer outputs. The resulting damping set was binned into $0.1 \%$ damping histograms (Figure 15). The damping histogram shows a peak at $0.4 \%$ damping, with about $5 \%$ of the modes below $0.3 \%$ damping. The baseline damping value was set at $0.3 \%$.

Optical Model: The optical ray trace models for AIA and HMI were provided by analysts at the Lockheed Martin Solar and Astrophysics Lab (LMSAL). A linear matrix was created to map the small optical motions to image motions measured on the CCD (e.g. LOS errors) using the ray trace models.

Control models: SDO features two control systems that influence the pointing of the spacecraft: (1) attitude control system (ACS) and (2) instrument stabilization system (ISS). The ACS consists of a standard proportional-integralderivative (PID) controller and a second-order lowpass filter for suppressing the response of low frequency flexible modes. For jitter analysis, the main purpose of the ACS is to stabilize the three rigid-body modes. It does not affect high frequency jitter motions. Both AIA and HMI have an ISS that further attenuates residual pointing errors around body $\mathrm{Y}$ and $\mathrm{Z}$ axes (or tip/tilt of the LOS vector) from the ACS. The ISS has much higher bandwidth than the ACS and does impact the jitter

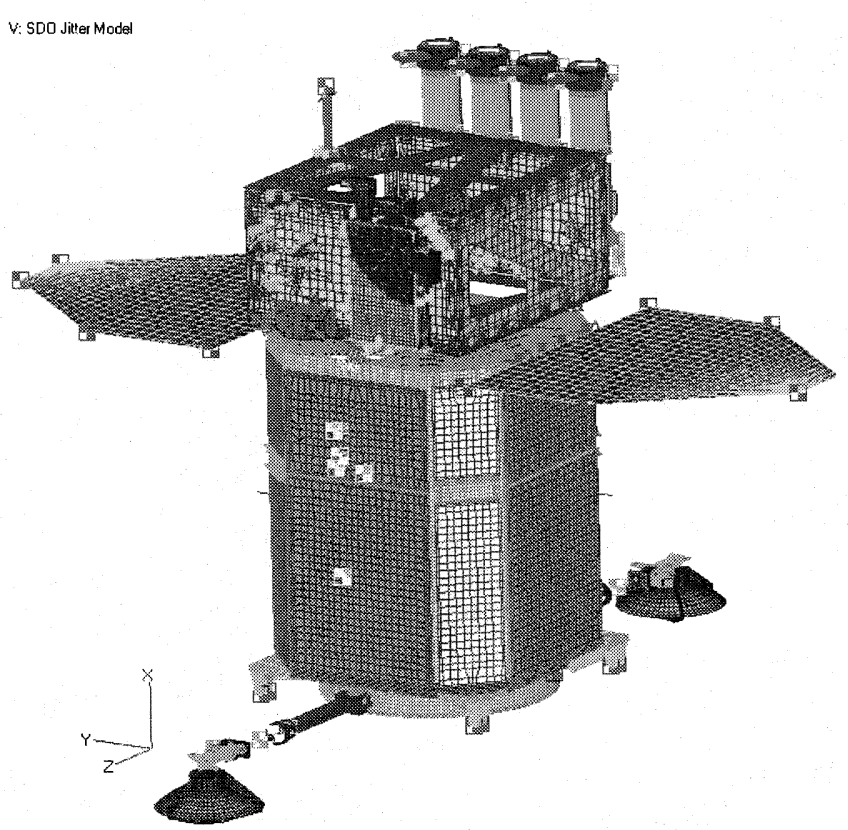

Figure 14 SDO jitter FEM

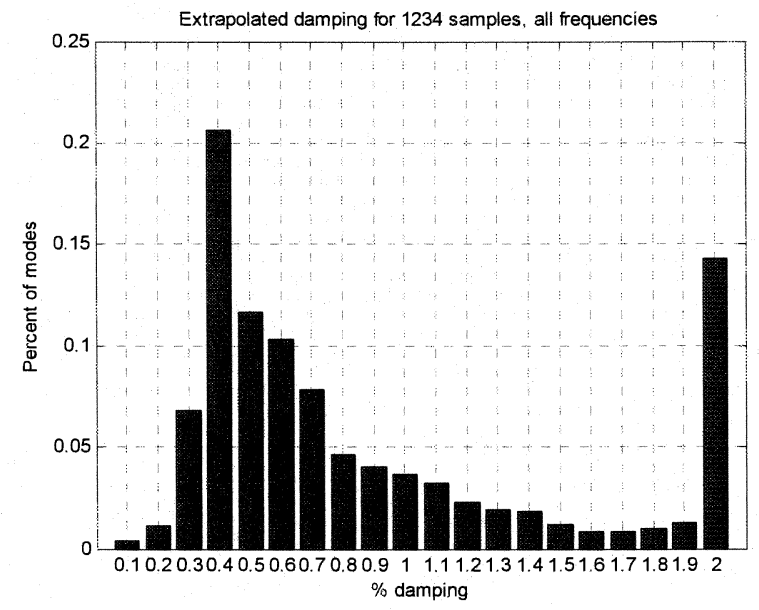

Figure 15 SDO jitter test damping histogram performance of the system. The disturbance rejection capabilities of each ISS were modeled as high-pass filters: the AIA model was a second order filter with a corner frequency at $14 \mathrm{~Hz}$, and the HMI model was a fourth order filter with a corner frequency at $45 \mathrm{~Hz}$. Both ISS models were verified against the instrument controller test data.

Uncertainty: There were two types of uncertainties considered in the SDO wheel jitter analysis. The first type was the modal gain uncertainty since the magnitudes of most of the high frequency modes were not validated by test data. To protect against the modal gain uncertainty, the jitter analysis team required a $100 \%$ margin on the current best estimate (CBE) jitter when compared to the allocated requirements. The percent margin requirement was defined as

$$
\% \text { margin requirement }=\left(\frac{\text { Requirement }}{\mathrm{CBE}}-1\right) * 100
$$

The second type of uncertainty was modal frequency. Only the frequencies of primary modes will be matched to the test data, and the frequencies of other modes could be in error by 5 to $10 \%$ or more. In order to estimate the impact of modal frequency uncertainty on the analysis results, the SDO jitter team performed frequency sensitivity analyses 
by either varying the FEM modes or the input disturbance frequencies by $+/-10 \%$. The worst-case result from the frequency sweep studies was reported as CBE to guard against frequency uncertainty.

\section{B. Analysis Approach}

The semi-analytical disturbance model, combining tonal and broadband noise is given by

$$
d_{j}(t)=\underbrace{\sum_{k=1}^{N_{j}} C_{j k} \Omega^{2} \sin \left(2 \pi h_{j k} \Omega t+\phi_{j k}\right)}_{\text {Tonal Disturbance }}+\underbrace{Q_{j}(t)}_{\text {Random Disturbance }}
$$

where all tonal disturbance parameters were defined in Section II and $Q_{j}$ is the broadband noise component for the $j^{\text {th }}$ disturbance.

As discussed in Section II-E, the forces in and torques about the $X$ axis are 90 deg out of phase with the disturbance forces in and moments about the $Y$ axis, so the phase terms can be written as:

$$
\phi_{2 k}=\phi_{1 k}+\pi / 2, \quad \phi_{5 k}=\phi_{4 k}+\pi / 2
$$

In addition, the radial forces and torques should share the same amplitude coefficient and harmonic numbers, i.e. $C_{l k}=C_{2 k}, C_{4 k}=C_{5 k}, h_{l k}=h_{2 k}$, and $h_{4 k}=h_{5 k}$. To perform the analysis, the phases $\phi_{j k}$ in Eq. (9) are modeled as uniform random variables, distributed between 0 and $2 \pi^{1}$. Assuming all random phases are uncorrelated and taking into account the phase relationship stated in Eq. (10), the PSD of the $i^{\text {th }}$ wheel disturbances $\left(S_{w i}\right)$ can be written as

$$
S_{w_{i}}(\omega)=\left[\begin{array}{ccccc}
S_{i 1} & S_{i 2} & 0 & & \\
-S_{i 2} & S_{i 1} & 0 & 0_{3 x 2} & \\
0 & 0 & S_{i 3} & & \\
& 0_{2 \times 3} & & S_{i 4} & S_{i 5} \\
& & & -S_{i 5} & S_{i 4}
\end{array}\right]+\left[\begin{array}{c}
S_{Q_{i 1}} \\
S_{Q_{i 2}} \\
S_{Q_{i 3}} \\
S_{Q_{i 4}} \\
S_{Q_{i 5}}
\end{array}\right],
$$

where

$$
\begin{gathered}
S_{i 1}=\sum_{k=1}^{N_{i 1}} \frac{C_{1 k}^{2}}{2} \pi\left[\delta\left(\omega+\omega_{1 k}\right)+\delta\left(\omega-\omega_{1 k}\right)\right], \quad S_{i 2}=\sum_{k=1}^{N_{i 1}} \frac{C_{1 k}^{2}}{2} \pi j\left[\delta\left(\omega+\omega_{1 k}\right)+\delta\left(\omega-\omega_{1 k}\right)\right] \\
S_{i 3}=\sum_{k=1}^{N_{i 3}} \frac{C_{3 k}^{2}}{2} \pi\left[\delta\left(\omega+\omega_{3 k}\right)+\delta\left(\omega-\omega_{3 k}\right)\right] \\
S_{i 4}=\sum_{k=1}^{N_{i 4}} \frac{C_{4 k}^{2}}{2} \pi\left[\delta\left(\omega+\omega_{4 k}\right)+\delta\left(\omega-\omega_{4 k}\right)\right], \quad S_{i 5}=\sum_{k=1}^{N_{i 4}} \frac{C_{1 k}^{2}}{2} \pi j\left[\delta\left(\omega+\omega_{4 k}\right)+\delta\left(\omega-\omega_{4 k}\right)\right]
\end{gathered}
$$

and $S_{Q_{i j}}$ is the PSD of broadband noise data for the $i^{\text {th }}$ wheel and the $j^{\text {th }}$ disturbance. Recall from Section II-E the broadband noise PSDs were obtained from the data directly after subtracting out the tonal disturbances. The frequencies $\omega_{j k}$ are equal to $2 \pi f_{i} h_{j k}$. Since there are four wheels on SDO, the PSD matrix for all wheel disturbance inputs is block diagonal and can be written as

$$
S_{w w}(\omega)=\left[\begin{array}{cccc}
S_{w_{1}} & & & 0 \\
& S_{w 2} & & \\
& & S_{w_{3}} & \\
0 & & & S_{w_{4}}
\end{array}\right],
$$

assuming the phases between wheels are also random and uncorrelated.

The output performance PSD matrix resulted from wheel disturbances is defined as ${ }^{5}$ 


$$
S_{z z}=G_{z w}(\omega) S_{w w}(\omega) G_{z w}^{H}(\omega)
$$

where $G_{z w}$ is the transfer function matrix that maps the wheel disturbance inputs to pointing performance $z$ (LOS jitter). The variance of each component output $z_{i}$ can be calculated by

$$
\sigma_{z_{i}}^{2}=\frac{1}{2 \pi} \int_{-\infty}^{\infty} G_{z_{i} w}(\omega) S_{w w}(\omega) G_{z_{i} w}^{H}(\omega) d \omega
$$

Using the above equation, the RMS pointing error from all wheel disturbances can be calculated without performing time-domain simulations. Furthermore, since the broadband noise also excites the wheel axial and whirl modes, an increased jitter could occur if one of the wheel structural modes coincides with the spacecraft structural mode. To protect against structural mode uncertainty, sensitivity analyses were performed by varying the input frequencies of the wheel disturbance PSD by $+/-10 \%$ and report the worst case jitter observed from the sensitivity analyses. The jitter predictions and various sensitivity analyses were performed using Matlab, Simulink, and Nightsky Systems Inc.'s Disturbance-Optics-Controls-Structures (DOCS) Toolbox.

\section{Analysis Results}

There are four AIA telescopes, each with two axes of performance outputs (tip and tilt of the LOS error) which result in a total of eight jitter responses. All eight were calculated and the largest was compared against the wheel jitter allocation. Figure 16 shows the AIA jitter due to each wheel disturbance component (radial force, axial force, radial moment, and broadband noise). The 70 mas allocation and the $100 \%$ margin requirement (35 mas) are also shown as red dashed and black dotted horizontal lines in the plot, respectively. At low wheel speeds ( $<400 \mathrm{RPM})$, the broadband noise dominate the jitter response, whereas at high wheel speed ( $>400 \mathrm{RPM})$, jitter responses due to tonal disturbances are significantly larger than those caused by the broadband noise.

After combining all the wheel disturbance sources, the final AIA jitter response is shown in Figure 17. The wheel jitter allocation is exceeded for wheel speeds greater than $500 \mathrm{RPM}$. The 100\% margin allocation can be met only if the wheel speeds are limited to 300 RPM. Since the broadband noise determines the jitter response of the telescope at low wheel speeds, it must be included in the jitter analysis.

For the HMI instrument, there are two performance outputs for the single telescope. The maximum jitter response over the two outputs was again computed and is reported here. Figure 18 and Figure 19 demonstrate that the HMI results follow similar trends as the AIA jitter results. However, the HMI jitter response exceeds the wheel $100 \%$ margin requirement even at very low wheel speeds.

Based on these results, the SDO team decided to conduct validation tests on the ground in order to understand the conservatism in the analysis before choosing the most appropriate wheel jitter mitigation strategy.

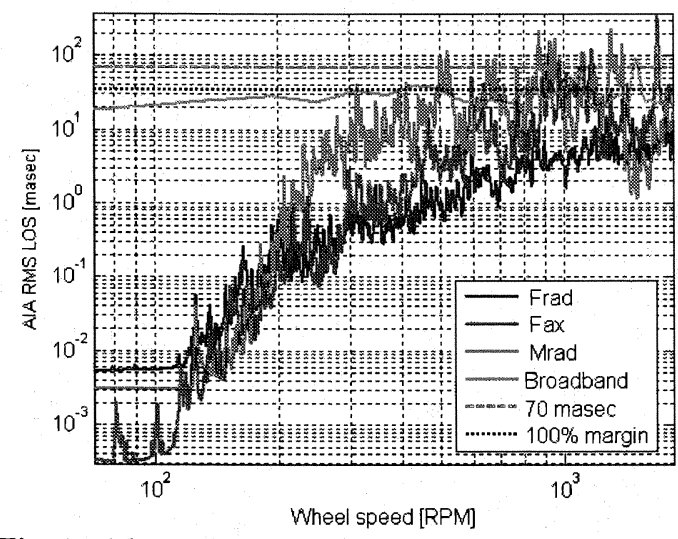

Figure 16 Maximum AIA telescope jitter due to different wheel disturbance components

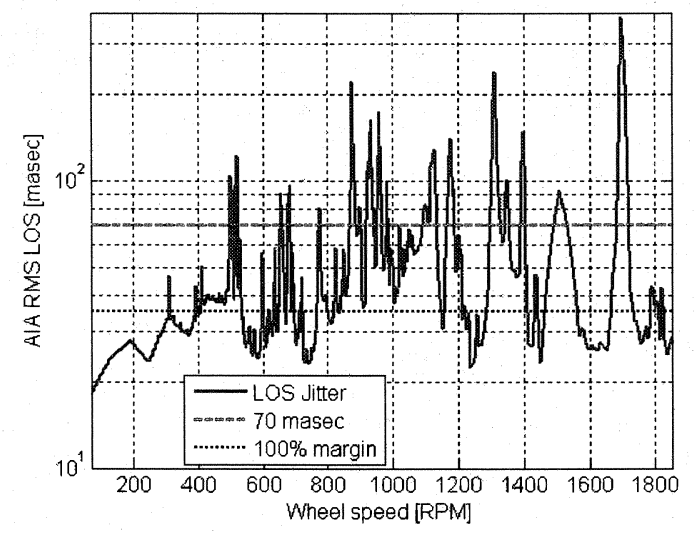

Figure 17 Maximum AIA telescope jitter due to all wheel disturbance components 


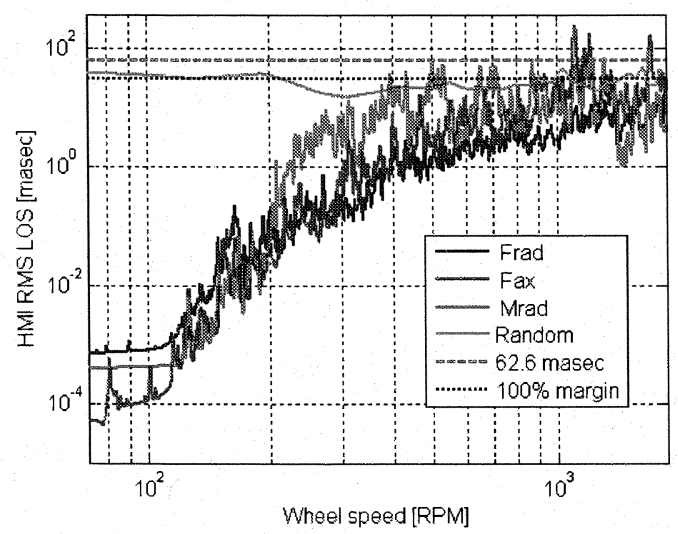

Figure 18 Maximum HMI telescope jitter due to different wheel disturbance components

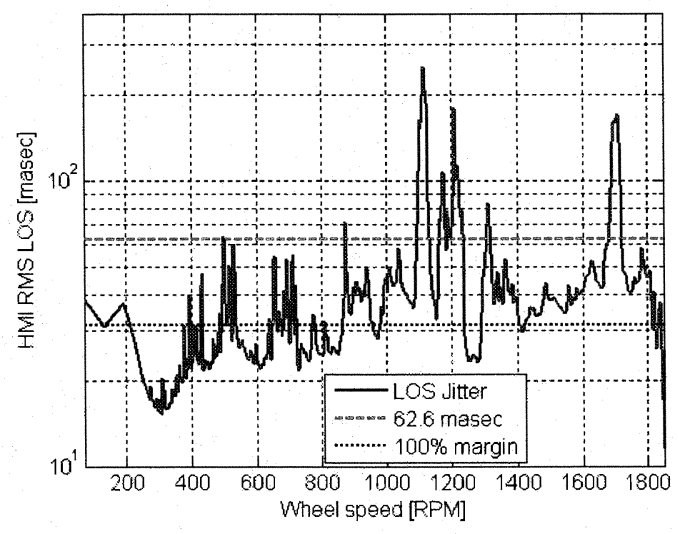

Figure 19 Maximum HMI telescope jitter due to all wheel disturbance components

\section{Ground Validation Tests and Results}

The reaction wheel jitter analysis process was validated by measuring the jitter response of the structure when excited by a wheel, and comparing that to a prediction created from a model of the ground test configuration. One of the flight wheels was mounted on the SDO Structural Verification Unit (SVU), which is a duplicate of the flight structure, with complete wheel electronic integration. A sketch of the setup is shown in Figure 20. The software for the wheel test incorporated a simple proportional-integrator controller developed to control the wheel speed using the wheel tachometer feedback. The control software was loaded to the SDO Attitude Control Electronics (ACE) engineering test unit (ETU). The wheel telemetry, including torque commands, motor current, and tachometer pulse counts, was monitored by the NTGSE and the ASIST workstation. The NTGSE is a Bus Controller Simulator (BCS) that provides the 1553 interface to the ACE. The ASIST workstation is a PC which, when used in conjunction with the BCS, simulates the Single Board Computer (SBC) and the ground station. This computer tool allowed the testers to interact with the flight software loaded in the ACE.

As the flight wheel excited the SVU structure, tri-axial accelerometers mounted at each of the four AIA telescopes, the HMI instrument, and the three bus-instrument module interface locations measured the jitter vibration levels. All the test data were logged using the DataPhysics system at a sample rate of $1024 \mathrm{~Hz}$. An additional tri-axial accelerometer was mounted at the flight wheel housing. The measured accelerations at this location provided a high quality wheel disturbance signal at a frequency equal to the wheel speed (e.g. the fundamental harmonic), which gave a more accurate indication of wheel speed than the tachometer.

During the tests, two operating modes were employed. First, the flight software commanded the wheel to ramp the speed at a fixed rate of acceleration. A slow acceleration of $0.1 \mathrm{RPM} / \mathrm{sec}$ was used to study the jitter at low wheel speeds, from zero to $700 \mathrm{RPM}$, while a faster acceleration rate of $1.0 \mathrm{RPM} / \mathrm{sec}$ was used to investigate jitter up to 2000 RPM (the faster acceleration was needed to shorten the duration of that portion of the test). Second, the flight software commanded the ACE to drive the wheel to a specific wheel speed and hold the speed constant for the duration of the dwell speed tests $(2-5 \mathrm{~min}$.) The ramp speed tests allowed the test engineers to identify problematic wheel speeds that corresponded to large jitter motions observed at the instruments. The test engineers then targeted those speed ranges using the dwell speed mode. 


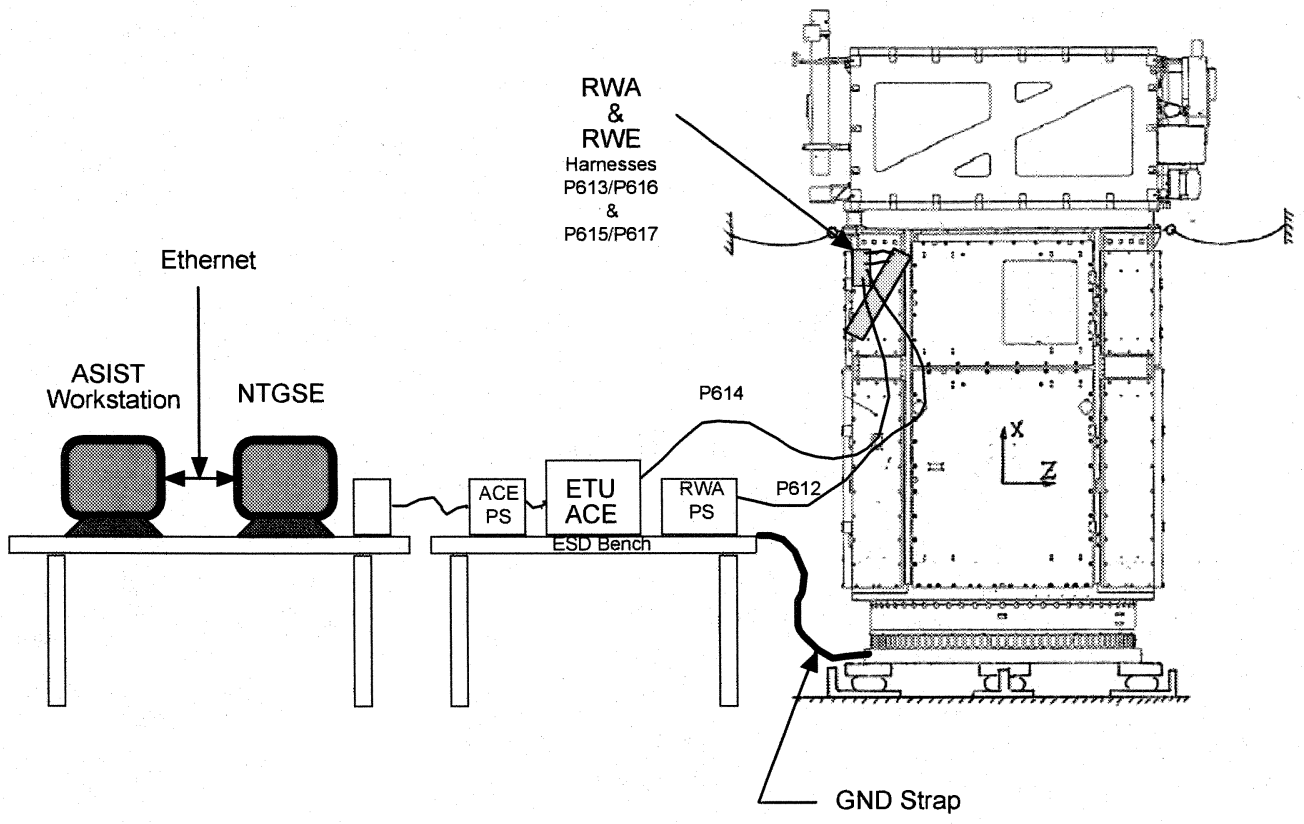

Figure 20 SVU and flight wheel jitter test setup

A representative set of the wheel ramp results are shown in Figure 21. The blue crosses represent the root-meansquare (RMS) acceleration data measured during the tests, the dashed line shows the analytical prediction using the disturbance model and analysis described above plus the background noise, and the solid constant line indicates the background noise level during the tests. The plots in the top row illustrate RMS accelerations at AIA telescope \#3 during the low acceleration $(0.1 \mathrm{RPM} / \mathrm{sec})$, ramp speed test. This test focused on measuring disturbances at low wheel speeds ( $<700 \mathrm{RPM})$ corresponding to the expected wheel speed operational range on orbit based on the jitter analysis results. From Figure 21 and other test data, the results show that our analytical prediction is generally a factor of 1.5 to 2.0 larger than the measured accelerations. Based on this observation, the required jitter analysis margin on the wheel disturbances was reduced from $100 \%$ to $66.7 \%$ (the allowable jitter was increased by a factor of 1.33). The plots on the bottom row of Figure 21 show the acceleration measurements from the same location during the high acceleration $(1.0 \mathrm{RPM} / \mathrm{sec})$ ramp speed test. At high wheel speeds ( $>700 \mathrm{RPM})$, the analysis prediction follows the measured data surprisingly well given the uncertainties in the structural FEM. The flight wheel plus SVU jitter tests validated the modeling and analysis approach used for SDO.
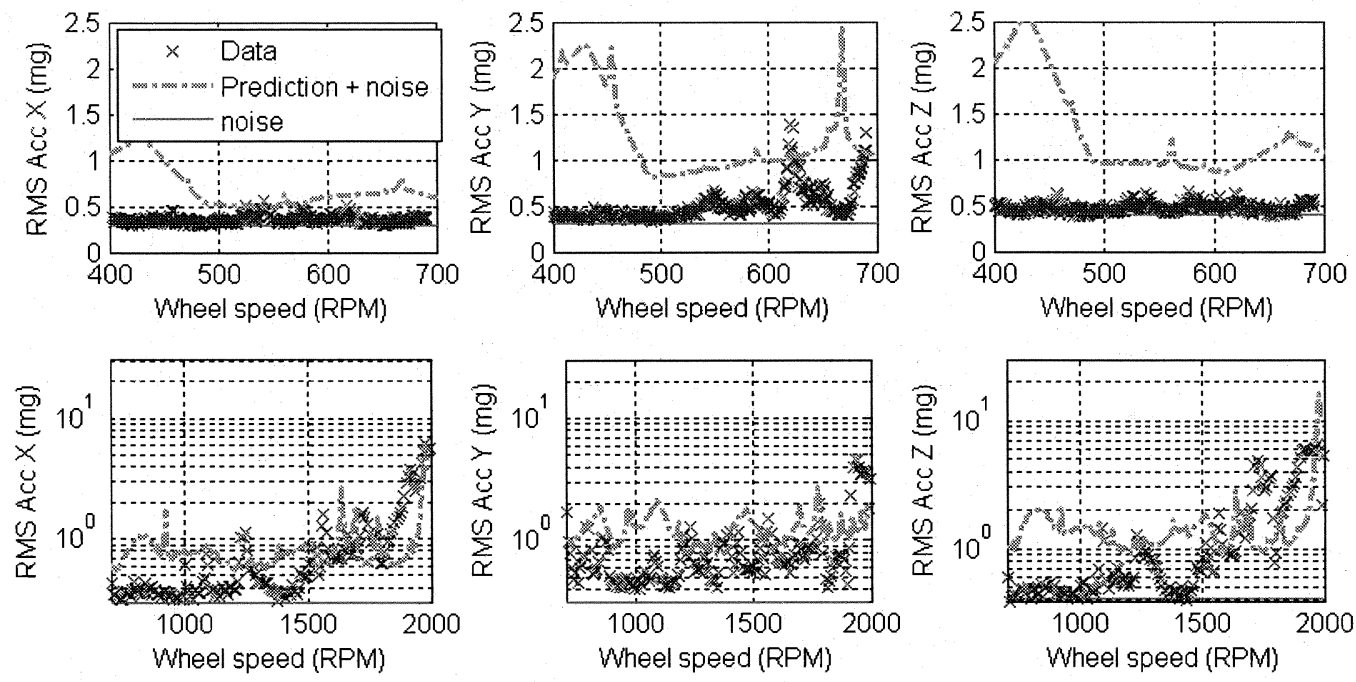

Figure 21 SVU wheel jitter results: (top) low wheel speed (bottom) high wheel speed 
By lowering the desired margin requirement from $100 \%$ to $66.7 \%$ based on the ground validation results, the maximum wheel speeds allowed to meet the $66.7 \%$ margin requirement is about 400 RPM for both AIA and HMI. Since SDO experiences small torques on-orbit, it is possible to run the wheels from -400 RPM to +400 RPM and still meet the four week momentum unload requirement. Therefore, the current operational plan that minimizes the wheel jitter is to limit the wheel speeds to within +/- 400 RPM during the science mode.

\section{On-orbit Validation Test Plan}

Although detailed analysis and assembly level tests were performed in order to obtain good jitter predictions, there were still several sources of uncertainties in the system. The structural finite element model will not have all the modes correlated to test data at high frequencies $(>50 \mathrm{~Hz})$. The performance of the instrument stabilization system will not be known exactly but should be close to the analytical model. A true disturbance-to-LOS observatory level test will not be available due to the tight schedule of the flight spacecraft, the cost in time and manpower, difficulties in creating gravity negation systems, and risks of damaging flight hardware. To protect the observatory jitter performance against model uncertainties, the jitter team has devised several on-orbit contingency plans in addition to reserve margins on analysis results.

As discussed in the previous section, the wheel speeds are nominally limited to 400 RPM (out of the $\sim 3000$ RPM momentum limit) in order to satisfy the wheel jitter requirement. This constraint will force the wheels to cross zero wheel speed and uses only a small portion of the allowed wheel speed range. Although analysis has shown that the jitter performance can be met during zero-wheel-speed crossings, and the Goodrich wheel has been shown to achieve its full life limit regardless of zero crossings, the SDO team would still like to avoid this situation in order to reduce risk. As a result, once SDO gets on orbit, the team plans to test the wheel-induced jitter, and will try to extend the wheel speed limit if possible to eliminate zero-speed crossings.

The basic methodology behind the on-orbit tests is to slew one wheel at a time in the speed range of interest while ensuring that the remaining three wheels stay inside the prescribed nominal range (e.g. +/- 400 RPM). Thus a large jitter response can be associated with the slewing wheel. For each test, the speed slew range is limited to 800 RPM since the other wheels must remain in the nominal range. The attitude control system's Delta-H and Science modes are used to bias the targeted wheel to a prescribed speed outside the nominal range, while the remaining three wheels are biased at either extreme of the nominal range. The spacecraft jitter is sensed via the two limb sensors within the AIA and the HMI instruments sampled at $256 \mathrm{~Hz}$. The results of the on-orbit tests will identify the true jitter-limited wheel speed range.

To minimize the impact on flight software, the on-orbit jitter algorithm was designed to be as simple as possible, and to minimize the effects of tachometer noise feedback to the system. The algorithm for the on-orbit wheel jitter tests is summarized in the following:

a) Implement a digital low pass filter to attenuate the high frequency noise in the measured wheel momentum signals from tachometers.

b) Compute the initial desired momentum bias in the body frame, and invoke the Delta-H and Science modes to take the wheel speeds from their current values to their desired values. It is assumed that the wheel speeds have reached steady-state prior to start of the wheel jitter test. SDO employs four reaction wheels in a pyramid configuration about the body $\mathrm{X}$ axis, i.e., there are two pairs of opposing wheels tilted away from the $\mathrm{X}$ axis towards $\mathrm{Y}$ and $\mathrm{Z}$ axes, respectively. For example, if we assume that the targeted wheel is wheel no. 1 , and it is desired to slew that wheel from an initial wheel speed of $w_{0}$ to $w_{0}+800$, then the initial wheel speeds for the other three wheels must be set to [400 -400 400] RPM, respectively. The negative speed corresponds to wheel no.3 which is the wheel that opposes wheel no. 1 .

d) Set the initial value of the slew torque commands to the bias values

$$
T_{\text {slew }}=T_{\text {bias }} * N_{w}
$$

where $T_{\text {bias }}$ represents the torque bias, and $N_{w}$ denotes the null vector in the wheel space. The variable $T_{\text {bias }}$ is chosen to provide a desired slew rate for the targeted wheel.

e) At every minor cycle (40 seconds), compute the difference in the current wheel momentum and the momentum in the previous cycle for the target wheel.

$$
w d=w h_{k}(i)-w h_{k-1}(i)
$$


where index "i" denotes the target wheel, the index " $\mathrm{k}$ " refers to the current cycle, and the index "k-1" refers to the previous cycle.

f) If $|w d|<=w d_{\text {lim }}$, apply a delta correction to the slew torque commands to increase the slew rate:

$$
T_{\text {slew }}=T_{\text {slew }}+T_{\text {corr }} * N_{v}
$$

where $T_{\text {corr }}$ represents the scale of the torque correction, and is computed as follows.

$$
T_{\text {corr }}=\alpha^{*} \operatorname{sgn}\left(T_{\text {bias }}\right) *|| w d\left|-w d_{\text {lim }}\right|
$$

where $\alpha$ is scale factor chosen at 0.025 .

g) Stop the experiment when either the target wheel speed reaches its final prescribed wheel speed, or the absolute value of the speed of one or more of the supporting wheels reaches the maximum allowed range.

Both low-pass filtering of the tachometer signals (step (a)) and 40 second interval for computing the wheel accelerations (step (e)) are methods recommended to reduce the effects of the tachometer noise.

The wheel slew algorithm was implemented within the high-fidelity simulation. This simulation includes detailed models of components and ACS modes, as well as structural dynamics. The flexible dynamics are represented by the finite element model normal modes. Figure and Figure 23 illustrate a simulation run with wheel no. 1 (facing $+Y$ side of spacecraft) as the targeted wheel. The wheel was slewed from 2200 RPM to just over 2500 RPM in about 1500 seconds. Here, the initial speeds for wheels no. 2-4 were chosen as [ $350-350$ 350] RPM, respectively. Note that these wheels remain in the allowable range during the course of the test. Figure 23 illustrates the LOS jitter at the CCD of the HMI instrument. Significant excursions, exceeding the HMI wheel allocation of 60 mas, are observed at wheel speeds of 2300-2400 RPM.

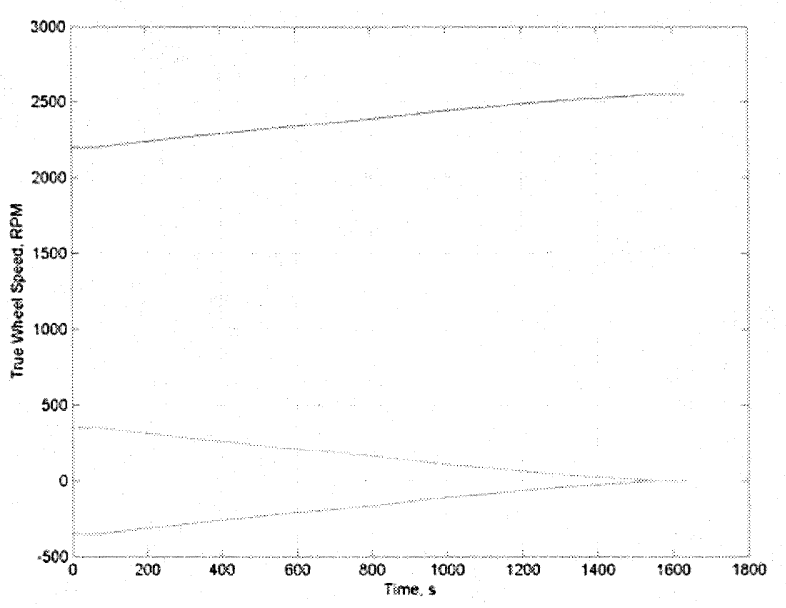

Figure 22 True wheel speeds

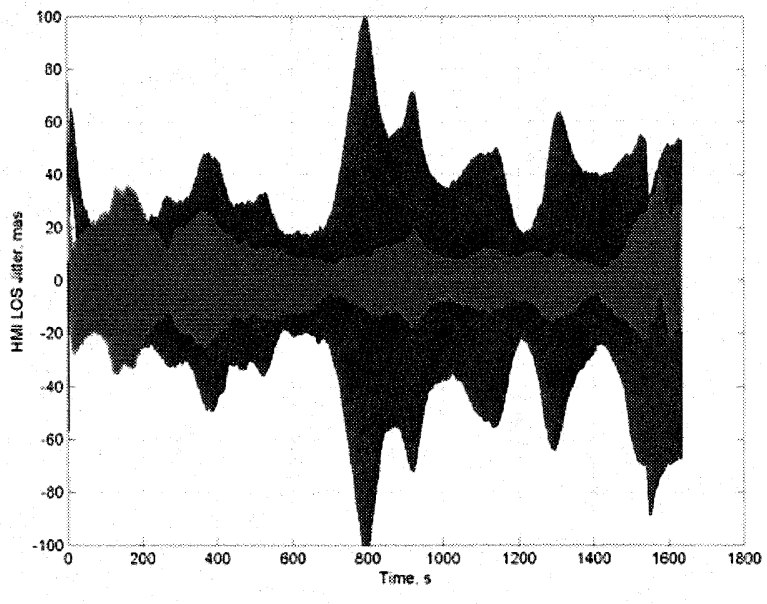

Figure 23 HMI line-of-sight jitter

\section{Concluding Remarks}

This paper describes the reaction wheel disturbance modeling and jitter analysis performed on SDO. It provides descriptions of the ground tests conducted for validating the wheel modeling and analysis approach. Jitter predictions based on the calibrated models indicate that SDO will have an acceptable level of jitter due to the reaction wheels if the wheel speeds are limited to +/- 400 RPM. In recognition of the fact that model uncertainties still exist even after extensive model correlation effort, the paper then lays out the jitter test plan to identify the true wheel speed limits during the commissioning phase of the mission.

The SDO jitter analysis highlights the potential importance of the wheel higher harmonic and broadband wheel disturbances. Traditionally only static and dynamic imbalance (i.e. fundamental harmonics) levels are specified in 
requirements. For sub-arcsecond pointing missions without isolation systems, the SDO team recommends that specifications for higher harmonics and broadband noise levels be developed in order to identify potential jitter problems. In cases where such higher order effects are predicted to result in unacceptable jitter, it is possible to limit the wheel speed (as in the case for SDO), place wheel speeds in a particular range by implementing a high sample rate jitter sensor ${ }^{6}$, or implement an isolation system to reduce the sensitivity to wheel higher harmonics. The approach chosen by SDO is the least risky and costly option since the torque buildup for SDO is expected to be small. The team expects the wheel speed limit can be expanded after the on-orbit jitter tests.

\section{Acknowledgments}

The authors would like to thank Thomas Kenney (SDO GNC lead) and Wendy Morgenstern (SDO ACS lead) for their assistance, guidance, and support for wheel verification and validation tests. The ground validation tests would not have been possible without the help of Pete Mule, William Haile, Brian Ross, Kirkland Brown, and SDO ACS, mechanical, and flight software teams.

\section{References}

${ }^{1}$ Gutierrez, H., "Performance Assessment and Enhancement of Precision Controlled Structures During Conceptual Design," Ph.D. Dissertation, MIT Department of Aeronautics and Astronautics, Cambridge, MA, 1999.

${ }^{2}$ Masterson, R., Miller, D., and Grogan, R., "Development and Validation of Reaction Wheel Disturbance Models: Empirical Model," AIAA Journal of Sound and Vibration, Vol. 249, Issue 3, 2002, pp. 575-598.

${ }^{3}$ Bialke, B., "A Compilation of Reaction Wheel Induced Spacecraft Disturbances," AAS Guidance and Control Conference, 1997.

${ }^{4}$ Liu, K-C., Kenney, T., Maghami, P., Mule, P., Blaurock, C., and Haile, W., "Jitter Test Program and On-Orbit Mitigation Strategies for Solar Dynamic Observatory," $20^{\text {th }}$ International Symposium on Space Flight Mechanics, NASA Goddard Space Flight Center, September 2007.

${ }^{5}$ Papoulis, A., Probability, Random Variables, and Stochastic Processes, $3^{\text {rd }}$ ed., McGraw-Hill Companies, 1991 , Chaps. 10.

${ }^{6}$ Miller, S., Kirchman, P., and Sudey, J., "Reaction Wheel Operational Impacts on the GOES-N Jitter Environment," AIAA Guidance, Navigation, and Control Conference, AIAA-6736, 2007. 